EPiC Series in Computing
Volume 70, 2020, Pages 208-216
$\begin{gathered}\text { Proceedings of the 12th International Conference } \\ \text { on Bioinformatics and Computational Biology }\end{gathered}$

\title{
Measurement of similarity in $C$. elegans healthspan using dynamic time warping on movement features
}

\author{
Arun G. Govindaswamy ${ }^{1}$, Wahhaj Farooq ${ }^{1}$, Yiyang Wang $^{1}$, Ilyas Ustun ${ }^{1}$, \\ Daniela Raicu ${ }^{1, a}$, Jacob Furst ${ }^{1, b}$, and Hongkyun Kim ${ }^{2}$ \\ 1 School of Computing, DePaul University, Chicago, IL, USA \\ ${ }^{a}$ draicu@cdm.depaul . edu, ${ }^{b}$ jfurst@cdm.depaul . edu \\ 2 Chicago Medical School, Rosalind Franklin University, North Chicago, IL, USA
}

\begin{abstract}
C. elegans is an ideal organism for modeling aging research due to their simple neural connectome and relatively short life. Current issues faced within aging research includes the decoupling of lifespan and healthspan. We propose a method to efficiently measure the healthspan of a nematode by considering healthspan as a comparable characteristic of the lifespan of a worm, where the lifespan is represented as a temporal sequence. We apply Dynamic Time Warping (DTW) so that the healthspan of any set of worms can be compared based on locomotion feature values' similarity over time. This technique allows us to compare the effects of various gene knockouts on the healthspan of the worm, such as daf2, in comparison to its wildtype N2. Results show that daf2 worm increases the lifespan of the worm, and using DTW to compare each feature as it changes over time, we can see that the proportion of its life that it stays in a healthy state also increases compared to $\mathrm{N} 2$ worms. To validate the results, we measure the time period a worm is healthy and the time period it is frail using a method called frailty threshold analysis. This allows us to determine the day a worm converts from being healthy to frail. We then compare the longevity of healthy state and frail state between worm types based on the duration of healthy state and frail state to see that daf2 in fact extends its healthy state since the proportion it stays in healthy state is greater than that of N2.
\end{abstract}

\section{Introduction}

As the global population increases, aging has become a major concern due to the associated decline in health. It can be characterized by the progressive loss of physiological and cognitive function [1]. This negatively impacts the quality of life during the later stages of life of an organism. In the last few decades, aging research has been focusing on extending the lifespan with an assumption that this extension of life would also extend healthspan and have a positive impact on health and overall quality of life $[2,3]$. However, there have been contradictory results showing that increasing lifespan may not increase quality of life of an organism [4]. In our study, we try to address this issue by implementing a technique to compare feature values in the lifespan, represented as temporal sequences, to determine the extension or decline in healthspan.

Q. Ding, O. Eulenstein and H. Al-Mubaid (eds.), BICOB 2020 (EPiC Series in Computing, vol. 70), 
Caenorhabditis elegans (C. elegans) is widely considered an excellent model for aging and agerelated research due to its simple neuronal structure and fully mapped connectome [5,6]. Many genes within the C. elegans connectome have been studied and linked to aging, including daf2 gene, which is part of the metabolic pathway coding for insulin-like growth factors in nematodes [7].

The effect of daf2 gene has been discovered to play a role in the extending the lifespan of the C. elegans species. The attenuation of daf2 increases the lifespan by almost two fold [8]. This makes $C$. elegans a good candidate for modeling aging since the role of daf2 and various other insulin/insulin-like growth factor signaling pathway (IIS) genes have been studied for their effects on lifespan in multiple species, including humans $[9,10]$. Various studies have been done on different genes, including those in the IIS signaling pathway, to try and determine whether this increase in longevity of lifespan also increases the healthspan.

Healthspan represents the condition or quality of life of an organism during a given period of its life and is measured in multiple different ways. Some studies use multiple physiological parameters over the course of a worm's life such as homeostasis in response to external stress, movement, lipofuscin accumulation, and pharyngeal pumping $[4,11]$. Others have done physiological assessments using movement features, such as maximum velocity. It has been shown that locomotion features are ageing dependent and can be a good measurement for healthspan measurement $[3,12,13]$.

The lifespan of a worm can be defined as the length of time a worm lives from adulthood to its death. We defined healthspan to be the period the worm spent in a healthy state and frailty to be the period of the worm spent in unhealthy state. We define healthy and unhealthy state based on a threshold of physical activity, where the threshold is the day at which a worm turns from healthy state to frail state, determined by the median values of each movement feature. The lifespan in all experiments were assessed in two ways: chronologically - defined as the absolute number of days in the life of the worm from day start of adulthood to death, and physiological - defined as the percentage of the maximal lifespan determined based on movement feature values, where maximal lifespan is the full duration a worm lived from adulthood to death.

In an effort to determine whether various locomotion features can be used to detect an increase or decrease in healthspan, we present a technique to compare the healthspan between organisms using dynamic time warping (DTW) to compare the movement features calculated from video recordings of the N2 wildtype and daf2 gene knockout worms over the entire lifespan of each worm. Previous work using DTW has been used to analyze the movement patterns of $C$. elegans $[14,15]$. Using DTW allows us to compare the effects that daf2 has on the locomotion feature over time and compares this trend with N2 over time. DTW algorithm has been used effectively to compare time series data, such as speech, human activity, and other types of temporal data to detect patterns [16].

This allows us to compare the movement features of worms over time to determine similarity or dissimilarity regardless of the length of the lifespan of each worm. Measuring healthspan as a temporal feature by comparing the locomotion features over time allows us to apply DTW since we compare the similarity of temporal sequences of varying length to see how similar or dissimilar they are between each other. This is significant since daf2 worms are known to last almost twice as long as N2 worms, the application of DTW allows us to see how similar or different the two features are over time between two worm types, regardless of the actual length of lifespan [17]. DTW was specifically applied in our analysis because it is designed to handle sequences of different lengths. 


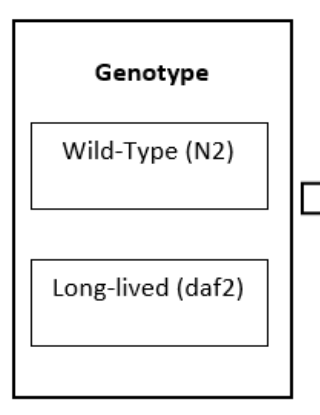

B

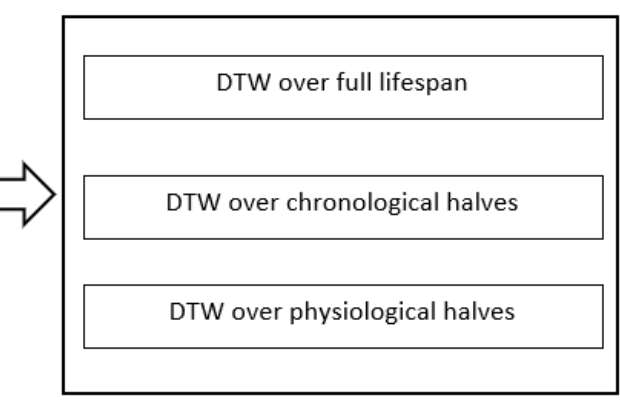

C

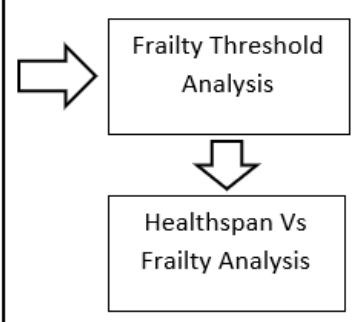

D

Figure 1: Experimental design of the study. (A) The data that was used in the study movement features of daf2 and N2 genotypes are used in the study. (B) The DTW experiment conducted to determine the similarity in the activity of daf2 and N2 worm and repeated three times over its varying lifespan. (C) The analysis of frailty threshold experiment conducted. (D) The healthspan versus frailty experiment conducted.

\section{Methods}

Our approach compares the healthspan of the N2 wildtype and the daf2 gene variant using locomotion features. We used dynamic time warping (DTW) to analyze the similarity in the movement features over the course of a worm's given life. DTW algorithm is used to compare these temporal sequences between the N2 wildtype and daf2 variant [17].

To determine when a worm has become frail, we performed a frailty threshold analysis, which is done to determine which day a worm hits frail state in its lifespan. Based on the cumulative results of this analysis, we were able compare the healthspan and lifespan of the worms to see whether daf2 not only extends the lifespan of a worm but whether it plays a significant role in health span extension. Figure 1 represents the diagram of our experimental design and analysis.

\subsection{Data}

Our work comprises of video and image data of the C. elegans worm, including N2 wildtype and daf2 genetic variant. The video data acquisition is based on our in-house built $C$. elegans tracking system [18]. The current video data is acquired and recorded by the tracker at varied frame rates measured in frames per second (fps), ranging from 8 fps to 39 fps with a spatial resolution of 1280 by 960 pixels. We recorded the movement of 10 wild type N2 and 10 daf2 worms. While the data initially consisted of 10 daf2 and 10 N2 worms, 4 daf2 and 2 N2 worms were removed as the worms were inconsistent with their respective mean lifespans.

After removal of any short lived worms, our data used movement features derived from 8 individual food deprived wild-type worm and 6 individual food deprived daf2 worm recorded every other day, starting from adulthood until their death. The video recorded from the tracker is saved as a series of images, which are then segmented to calculate the worm's centroid location within each frame. These centroids are then used to calculate the movement features between frames. The main features that we used to best represent healthspan markers are 
speed, measured in $\mu \mathrm{m} /$ second, acceleration, measured in $\mu \mathrm{m} /$ second $^{2}$, distance traveled, in $\mu \mathrm{m} /$ frame, and cell occupancy, measured as unique-cells-visited/minute. These features were used as health markers in the study to determine healthspan over time. Speed, acceleration, and cell occupancy were measured in physical time, whereas distance traveled is measured in video time. Cell occupancy is also measured in video space, which has a constant spatial resolution. Table 1 summarizes the video data in the study. This table shows the range for frame rate between each worm video within the same subset as well as the range for length of each video.

\begin{tabular}{|c|c|c|c|c|}
\hline $\begin{array}{c}\text { Worm } \\
\text { Type }\end{array}$ & $\begin{array}{c}\text { Worm } \\
\text { Number }\end{array}$ & $\begin{array}{c}\text { Total Number } \\
\text { of Days }\end{array}$ & $\begin{array}{c}\text { Range of } \\
\text { FPS }\end{array}$ & $\begin{array}{c}\text { Range of } \\
\text { Video Length }\end{array}$ \\
\hline daf2 & 2 & 25 & $8-31$ & $00: 20: 27-01: 14: 28$ \\
daf2 & 3 & 33 & $8-37$ & $00: 21: 01-00: 57: 17$ \\
daf2 & 4 & 31 & $21-31$ & $00: 04: 31-00: 34: 57$ \\
daf2 & 6 & 19 & $8-32$ & $00: 25: 03-00: 36: 48$ \\
daf2 & 7 & 23 & $27-38$ & $00: 20: 23-00: 37: 45$ \\
daf2 & 10 & 25 & $21-29$ & $00: 22: 01-00: 39: 10$ \\
\hline N2 & 1 & 17 & $25-28$ & $00: 20: 24-00: 35: 41$ \\
N2 & 2 & 15 & $26-31$ & $00: 05: 35-00: 35: 00$ \\
N2 & 3 & 19 & $8-39$ & $00: 20: 32-00: 39: 39$ \\
N2 & 5 & 9 & $23-28$ & $00: 29: 50-00: 38: 39$ \\
N2 & 6 & 11 & $8-31$ & $00: 21: 10-00: 41: 05$ \\
N2 & 8 & 19 & $22-27$ & $00: 20: 10-00: 36: 25$ \\
N2 & 9 & 17 & $22-39$ & $00: 20: 36-00: 38: 31$ \\
N2 & 10 & 19 & $21-38$ & $00: 26: 39-00: 38: 57$ \\
\hline
\end{tabular}

Table 1: Summary of video data organized by gene type, worm number, total number of days recorded, range of frame rates for each video recorded, and the length of each video.

\subsection{Selecting threshold for frail state}

Threshold value is the median value for any given feature with falls below a certain percentage of the maximum feature value. Using the $\mathrm{N} 2$ worm as control, we determined a threshold value or the percentage of the maximal capacity of a movement feature as a cutoff a worm's feature values have to reach in order to mark that given day as the day the worm entered the frail state [4]. The values for each feature at day 1 represent the full functional capacity of a worm, which makes the locomotion activity on day 1 to be the maximum functional capacity possible for the worms. The key assumption here is the first 10 days are considered the first half of N2's lifespan (assumed to be our healthy period) and the last 10 days are considered the second half of the lifespan (assumed to be our frailty period). The frailty period of the genotype is defined as the day in which all the individual worms in the genotype entered the frail state. By this definition, the frail state and healthspan of the daf2 mutant was calculated. The median value of all movement activity on day 1 was measured for all the features: Speed $=403.40 \mathrm{\mu m} / \mathrm{second}$, Acceleration $=135.96 \mu \mathrm{m} /$ second $^{2}$, Distance Traveled $=11.97 \mu \mathrm{m} /$ frame, and Cell Occupancy $=31$ unique cells visited $/$ minute . 


\subsection{Measuring similarity in activity between daf2 and N2 using DTW}

DTW was performed pairwise over the set of all worms which includes 8 N2 worms, and 6 daf2 worms. Therefore, DTW was performed 24 times [(8 N2 worms x 6 daf2 worms $) / 2]$. There were 4 movement features used, so we had 4 channels of DTW being measured. The distance values calculated from DTW between any two given feature represented as a temporal sequence were collected and categorized into two sets - one set consists of the DTW results performed between two different genotypes or inter-genotype (e.g.: daf2-1 vs N2-1, N2-4 vs daf2-5) and the other set consists of the DTW results performed within the same genotype, or intra-genotype (e.g.: daf2-2 vs daf2-3, N2-1 vs N2-5). The experiment was repeated for first half and second half of the worm's life. The first and second half of the worm's life was measured both chronologically and physiologically.

To test for significance for our results, Mann-Whitney statistical test was performed on the two sets of results from DTW, intra- and inter-genotype distances. We used this significance test since the sample was small and non-normally distributed [19]. The p-values of this test were used to tell us whether the DTW results were due to the effects of the dependent variable, genotype, or due to chance.

\section{$3 \quad$ Results}

Our study analyzes the locomotion activity in the daf2 and N2 worms and compares the distribution of these features over their lifespan. Dynamic Time Warping (DTW) was used to compare the difference in their activity and the results shows that the locomotion feature values vary over time differently for daf2 than N2 - they have dissimilar trends in their features over time. The results suggest that the decline in the activity of daf2 is slower than the wild-type N2 genotype. Zhang et al. also showed lower physiological decline in the long-lived mutants than in the wild-type or short-lived mutants [20]. It was clear from the results of DTW that the daf2 and N2 genotypes have differences/dissimilarities in their movement activity. Based on the p-values from the Mann-Whitney test, we can reject our null hypothesis which states that the trend in movement feature values of daf2 and N2 are similar. If they were similar, then daf2 would not have an effect in increasing healthspan, which is not the case. It can be concluded from the DTW test that the movement activity of the daf2 and N2 genotypes are dissimilar. and these difference are statistically significant(all features: $\mathrm{p}<0.05,95 \%$ confidence interval).

We further analyzed the effect of lifespan extension by looking at the proportional change in healthspan. Four possible outcomes were analyzed - no effect on the healthspan, no effect on the frailty, compression of the period of frailty, and impact on both healthspan and frailty [21]. Frailty threshold analysis was introduced to classify the life of the worm as healthy and frail which we call the healthspan and frailty period [22]. The frailty period of the genotype is defined as the day in which all the individual N2 worms entered frail state, and stayed frail until their death. N2 worms entered frail state after spending at least $50 \%$ of its time in healthy state. When compared with the daf2 variant, the long-lived mutant spent its overall life healthier than the wild-type mutant. The long-lived mutant shows an increase in healthspan when measured both chronologically and physiologically. The results are consistent with Rollins et al. where they showed that daf2 enjoys an improved quality of life over the N2 genotype [3]. Hahm et al. also shows that the long-lived mutant daf2 enjoys a better quality of life than the wild-type mutant [12]. It can then be stated that using DTW algorithm allows us to measure and classify a worm's healthspan as similar or dissimilar to N2, regardless of the variation in each worm's lifespan. 


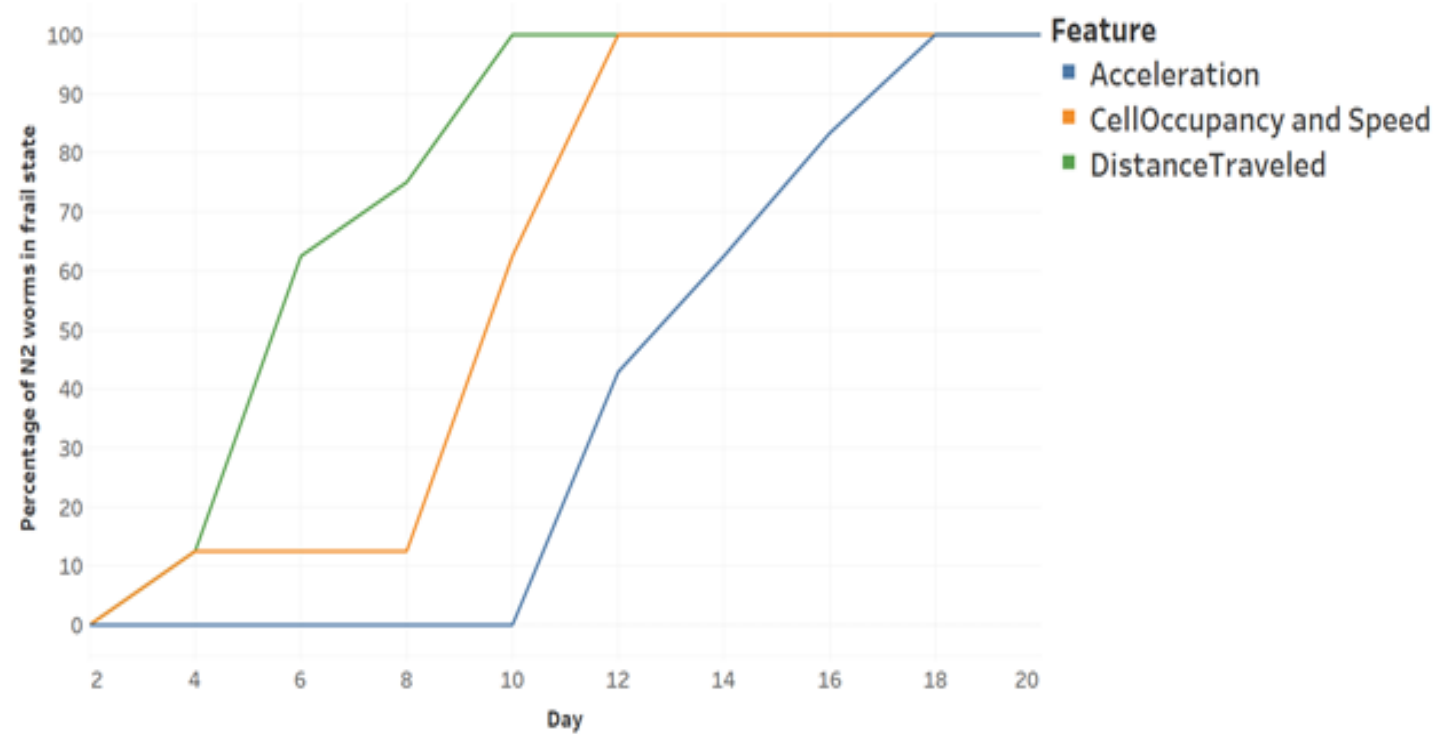

Figure 2: With a threshold of $25 \%$, the health state of N2 worms was recorded every other day. The graph shows the percentage of N2 worms in frail state on a given day when the threshold of $25 \%$ was used. Note that the cell occupancy and speed have similar curves and hence, only one curve is shown in the graph.

Our outcomes were consistent with three of the healthspan biomarkers - speed, acceleration and cell occupancy. With the distance traveled measure, though the daf2 variant shows an increase in healthspan when measured chronologically, the daf2 mutant doesn't seem to increase the healthspan. This result for distance traveled is consistent with the expected results for this feature $[4,20]$. It is important to note that the different trend that we see in distance traveled could also be due to the method of measurement since distance traveled was measured using video time, whereas all other features were measured in physical time. With other measures of health, the daf2 variant shows an increase and healthspan when measured chronologically.

\subsection{Frailty Threshold Analysis}

To determine the most optimal threshold value for frailty, we analyzed various threshold values ranging from $5 \%-100 \%$ of maximum capacity for a given feature, incrementing by $5 \%$ in each iteration. Using these proportions, we were able to determine the state that a worm was in during its lifespan: healthy or frail if its corresponding feature values hit the current threshold. Similarly, the onset value for all the other features were calculated and any activity less than the onset value was considered to be frail.

$50 \%$ of the life of $\mathrm{N} 2$ was in healthy state and the next $50 \%$ was in frail state when we used a threshold of $25 \%$. Hahm et al. used a threshold of $50 \%$ on its maximum functional capacity which in our case led all the $\mathrm{N} 2$ worms to enter frail state after day 2 when measured using the distance traveled health marker [12]. After a series of experiments, we chose a threshold of $25 \%$ as it was indicating at least half the life of the genotype N2 in healthy and frail state, which matched our assumption. Figure 2 displays the percentage of N2 worms which reach frail state 

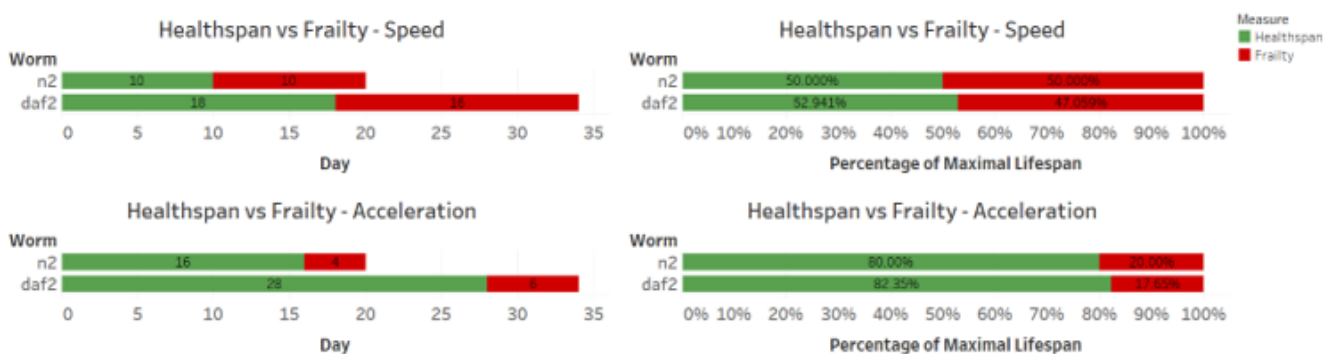

Healthspan vs Frailty - Distance Travelled
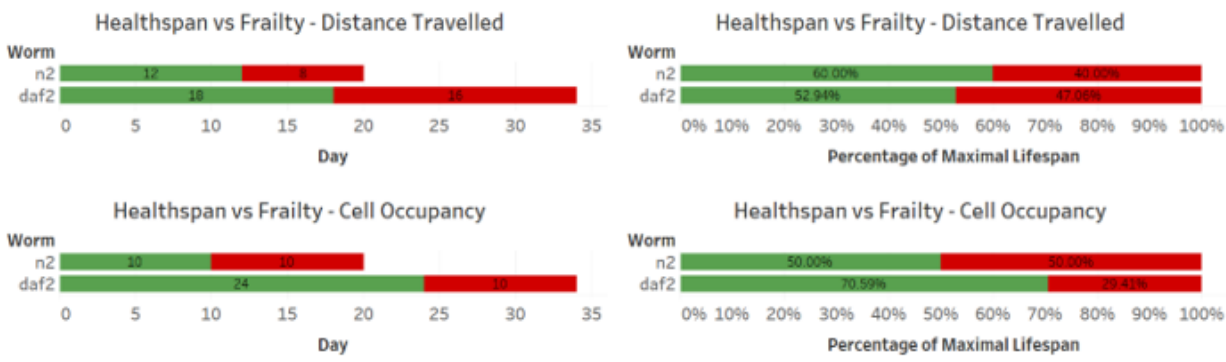

Figure 3: The bar graphs show the comparison of healthspan period and frailty period as a function of days (chronological) and lifespan (physiological).

using a threshold of $25 \%$ for each feature. Using this threshold, all the worms enters frailty on recorded day 6 . The frailty onset values for all healthspan biomarkers calculated using the $25 \%$ threshold are as follows: Speed $=100 \mu \mathrm{m} /$ second, Acceleration $=34 \mu \mathrm{m} /$ second $^{2}$, Distance Traveled $=3 \mu \mathrm{m} /$ frame, and Cell Occupancy $=7$ unique cells visited $/ \mathrm{minute}$.

\subsection{Healthspan Vs. Frailty}

Using our definition of healthspan and frailty, we analyzed the health of the daf2 worms. Figure 3 compares the healthspan and frailty over its lifespan. The frail state of individual worms in the daf2 genotype was analyzed using the onset value and categorized either as healthy or frail. The gene daf2 was considered frail on the day when all the individual worm in the genotype entered frail state. By this analysis, we found that the long-lived daf2 mutants extend both the healthspan and frailty period. When measured physiologically, daf2 worms seem to spend more time in healthy state than the wild-type gene. Even when the healthspan is measured chronologically, the daf2 worm seems to have extended their number of days in healthy state over the wild-type mutant.

When we only look at the extended days chronologically, the increase in healthy days seems to be more than the increase in unhealthy days. However, there is an exception in the case of one of our biomarkers - distance traveled. Although the healthspan seems to increase chronologically, daf2 has less healthspan than N2 when compared physiologically. Also, the extension of life has resulted in a greater number of days in frailty than in healthy condition. However, it is clear from our findings that the lifespan extension caused by daf2 influences the healthspan of the gene and results in extended period of healthspan. 


\section{Conclusion}

Contemporary aging research focuses on extending lifespan with a premise that it would also increase the healthspan of the individual. C. elegans, a model organism in aging research, has been analyzed to study the relationship between lifespan and healthspan. We know that the age associated decline comes with an expensive healthcare expenses and decreased quality of life in the later stages of life. Ideally, these effects of health decline because of age should be decreased. We proposed a simple approach to measuring the effect a gene has on the longevity of healthspan using DTW algorithm to compare locomotion feature as they change over time. By comparing the feature values over the course of the lifespan, we can measure the similarity between two lifespan curves to determine whether the gene in question either extends or reduces the healthspan of an organism.

Our findings using our method of healthspan measurement clearly show the impact of daf2 on healthspan and that the healthspan scales with lifespan. The number of healthy days of the worm increased in the long-lived mutant when measured chronologically and physiologically. Although the increase in the duration of healthspan is a beneficial outcome of daf2 knockout, we also expected the frail period to be compressed or remain the same. Contrary to our expectation, the extension of life has increased the frail period as well. This leaves us with the understanding that more research must go in extending the healthspan along with reducing the frailty of the worm. Further analysis of different genetic variants shown to effect healthspan will have to be performed to expand our understanding of their effect on frailty.

\section{References}

[1] Laura A. Herndon, Peter J. Schmeissner, Justyna M. Dudaronek, Paula A. Brown, Kristin M. Listner, Yuko Sakano, Marie C. Paupard, David H. Hall, and Monica Driscoll. Stochastic and genetic factors influence tissue-specific decline in ageing c. elegans. Nature, 419(6909):808-814, 2002.

[2] Carlos López-Otín, Maria A. Blasco, Linda Partridge, Manuel Serrano, and Guido Kroemer. The hallmarks of aging. Cell, 153(6):1194-1217, 2013.

[3] Jarod A. Rollins, Amber C. Howard, Sarah K. Dobbins, Elsie H. Washburn, and Aric N. Rogers. Assessing health span in caenorhabditis elegans: Lessons from short-lived mutants. The Journals of Gerontology: Series A, 72(4):473-480, Feb 2017.

[4] Ankita Bansal, Lihua Julie Zhu, Kelvin Yen, and Heidi A. Tissenbaum. Uncoupling lifespan and healthspan in caenorhabditis elegans longevity mutants. Proceedings of the National Academy of Sciences of the United States of America, 112 3:E277-86, 2015.

[5] Hildegard I.d. Mack, Thomas Heimbucher, and Coleen T. Murphy. The nematode caenorhabditis elegans as a model for aging research. Drug Discovery Today: Disease Models, 27:3-13, 2018.

[6] Michele Sammut, Steven J. Cook, Ken C. Q. Nguyen, Terry Felton, David H. Hall, Scott W. Emmons, Richard J. Poole, and Arantza Barrios. Glia-derived neurons are required for sex-specific learning in c. elegans. Nature, 526(7573):385-390, Oct 2015.

[7] Cynthia J. Kenyon. The genetics of ageing. Nature, 464(7288):504-512, 2010.

[8] Cynthia Kenyon, Jean Chang, Erin Gensch, Adam Rudner, and Ramon Tabtiang. A c. elegans mutant that lives twice as long as wild type. Nature, 366(6454):461-464, 1993.

[9] Andrzej Bartke. Impact of reduced insulin-like growth factor-1/insulin signaling on aging in mammals: novel findings. Aging Cell, 7(3):285-290, 2008.

[10] Ozlem Altintas, Sangsoon Park, and Seung-Jae V. Lee. The role of insulin/igf-1 signaling in the longevity of model invertebrates, c. elegans and d. melanogaster. BMB Reports, 49(2):81-92, 2016. 
[11] Katie Podshivalova, Rex A. Kerr, and Cynthia Kenyon. How a mutation that slows aging can also disproportionately extend end-of-life decrepitude. Cell Reports, 19(3):441-450, 2017.

[12] Jeong-Hoon Hahm, Sunhee Kim, Race Diloreto, Cheng Shi, Seung-Jae V. Lee, Coleen T. Murphy, and Hong Gil Nam. C. elegans maximum velocity correlates with healthspan and is maintained in worms with an insulin receptor mutation. Nature Communications, 6(1), 2015.

[13] Breanne L. Newell Stamper, James R. Cypser, Katerina Kechris, David Alan Kitzenberg, Patricia M. Tedesco, and Thomas E. Johnson. Movement decline across lifespan ofcaenorhabditis elegansmutants in the insulin/insulin-like signaling pathway. Aging Cell, 17(1), Jul 2017.

[14] Yiyang Wang, Carleton Smith, Mingfei Shao, Li Huang, Jacob Furst, Daniela Raicu, and Hongkyun Kim. C. elegans search behavior analysis using multivariate dynamic time warping. 2016 IEEE International Conference on Bioinformatics and Biomedicine (BIBM), 2016.

[15] Li Huang, Hongkyun Kim, Jacob Furst, and Daniela Raicu. A run-length encoding approach for path analysis ofc. eleganssearch behavior. Computational and Mathematical Methods in Medicine, 2016:1-9, 2016.

[16] Donald Berndt and James Clifford. Using dynamic time warping to find patterns in time series. AAAI Workshop on Knowledge Discovery in Databases, page 359-370, 1994.

[17] Stan Salvador and Philip Chan. Toward accurate dynamic time warping in linear time and space. Intelligent Data Analysis, 11(5):561-580, Oct 2007.

[18] Kyle Moy, Weiyu Li, Huu Phuoc Tran, Valerie Simonis, Evan Story, Christopher Brandon, Jacob Furst, Daniela Raicu, and Hongkyun Kim. Computational methods for tracking, quantitative assessment, and visualization of c. elegans locomotory behavior. Plos One, 10(12), 2015.

[19] Nadim Nachar. The mann-whitney u: A test for assessing whether two independent samples come from the same distribution. Tutorials in Quantitative Methods for Psychology, 4(1):13-20, Jan 2008.

[20] William B. Zhang, Drew B. Sinha, William E. Pittman, Erik Hvatum, Nicholas Stroustrup, and Zachary Pincus. Extended twilight among isogenic c. elegans causes a disproportionate scaling between lifespan and health. Cell Systems, 3(4), 2016.

[21] Malene Hansen and Brian K. Kennedy. Does longer lifespan mean longer healthspan? Trends in Cell Biology, 26(8):565-568, 2016.

[22] Malene Hansen, Stefan Taubert, Douglas Crawford, Nataliya Libina, Seung-Jae Lee, and Cynthia Kenyon. Lifespan extension by conditions that inhibit translation in caenorhabditis elegans. Aging Cell, 6(1):95-110, 2007. 